\title{
Effects of rhein lysinate on D-galactose-induced aging mice
}

\author{
YONG-ZHAN ZHEN ${ }^{1 *}$, YA-JUN LIN $^{2 *}$, KAI-JI LI ${ }^{3}$, GUANG-LING ZHANG ${ }^{1}$, \\ YU-FANG ZHAO ${ }^{1}$, MEI-MEI WANG ${ }^{1}$, JING-BO WEI $^{1}$, JIE WEI $^{2}$ and GANG HU ${ }^{2}$
}

\begin{abstract}
${ }^{1}$ Hebei Key Laboratory for Chronic Diseases, Tangshan Key Laboratory for Preclinical and Basic Research on Chronic Diseases, School of Basic Medical Sciences, North China University of Science and Technology, Tangshan, Hebei 063000; ${ }^{2}$ Key Laboratory of Geriatrics, Beijing Hospital and Beijing Institute of Geriatrics, Ministry of Health, Beijing 100730; ${ }^{3}$ Department of Histology and Embryology, Basic Medical College of Hebei United University, Tangshan, Hebei 063000, P.R. China
\end{abstract}

Received September 1, 2014; Accepted July 14, 2015

DOI: $10.3892 / \mathrm{etm} .2015 .2858$

\begin{abstract}
The aim of the present study was to investigate the anti-aging effects of rhein lysinate (RHL), and to explore its mechanism of action in a D-galactose-induced aging mouse model. Aging was induced by D-galactose $(100 \mathrm{mg} / \mathrm{kg} / \mathrm{day})$ that was subcutaneously injected to animals for 8 weeks. RHL was simultaneously administered once a day by intragastric gavage. The appetite, mental condition, body weight and organ index of the mice were monitored. Superoxide dismutase (SOD) and glutathione peroxidase (GSH-Px) activities were determined, and the levels of malondialdehyde (MDA) in the liver, kidney and serum were measured by appropriate assay kits. Western blot analysis was used to detect proteins associated with age. The results indicated that RHL may improve the appetite, mental state and organ conditions of the model mice, improve the activities of SOD and GSH-Px, reduce MDA levels and modulate the expression of age-associated proteins (Sirtuin 1, p21 and p16) in D-galactose-induced mice. Therefore, RHL may be effective at suppressing the aging process through a combination of enhancing antioxidant activity, scavenging free radicals and modulating aging-associated gene expression.
\end{abstract}

\section{Introduction}

Aging is not only a natural phenomenon which is an inevitable biological process, but is also associated with diverse chronic diseases, including cancer, Parkinson's and cardiovascular

Correspondence to: Professor Gang Hu, Key Laboratory of Geriatrics, Beijing Hospital and Beijing Institute of Geriatrics, Ministry of Health, 1 Dahua Road, Beijing 100730, P.R. China E-mail: huganglys2010@126.com

*Contributed equally

Key words: rhein lysinate, anti-aging, D-galactose, oxidative stress, antioxidant diseases $(1,2)$. With the increasing elderly population in the world, aging has already become an important public issue (3). Oxidative stress is considered to be significant in the pathophysiology of various diseases, including the aging process. Reactive oxygen species (ROS) damage cellular lipids, proteins and DNA, inhibiting their normal functions. An excess of ROS can result in cell aging and death $(1,2,4)$. Numerous studies have discovered that oxidative stress occurs during the pathogenesis of age-associated diseases (4-8).

D-galactose (D-gal)-treated mice have been demonstrated to display similar symptoms to those aging naturally (9). D-gal injection has since been widely used to establish a model for anti-aging research (10-13). D-gal, a reducing sugar, is a naturally occurring substance in the body, which is completely metabolized at normal concentrations. However, at higher concentrations it is converted to aldose, hydrogen peroxide and galactose oxidase, thus speeding up the generation of superoxide anion and oxygen-derived free radicals that impair the function of macromolecules and cells (14). Various studies have indicated that D-gal accelerates aging in mice, rats, houseflies and human fetal lung fibroblast and has been used as an aging model since 1985 (15). Studies with D-gal-induced mice have shown that D-gal-induced oxidative stress causes cognitive impairment, neurotoxicity, tissue injury and inflammation $(16,17)$.

The use of herbal medicines has increased globally due to their lower adverse effects, price and good efficacy in the majority of human illnesses $(18,19)$. In recent years, numerous traditional Chinese medicines have been found to possess potent anti-aging activities and have attracted considerable interest as potential candidates for the development of novel anti-aging therapies $(20,21)$. Rhein, one of the major bioactive constituents of the rhizome of rhubarb (Rheum palmatum Linn. or R. tanguticum Maxim), is a widely used traditional Chinese herb with broad pharmacological effects, including antidiabetic activity $(22,23)$, anti-inflammation and inhibition of interleukin-1-induced chondrocyte activation (24). However, due to its water insolubility, the efficacy of rhein is limited in vivo. Rhein lysinate (RHL) is the salt of lysine and rhein that is water soluble and so may be administered in vivo in drinking water.

A previous study from our laboratory demonstrated that RHL possessed an anti-aging effect in vitro, which may be 
associated with its anti-oxidative properties (25). However, no studies to date have addressed the effect of RHL on the aging process in vivo. The aim of the present study was, therefore, to use of the D-gal-induced aging model mice in order to investigate the anti-aging effects of RHL in vivo and explore the underlying anti-aging molecular mechanisms.

\section{Materials and methods}

Chemicals and reagents. Rhein (purity, 98\%) was purchased from Nanjing Qingze Medicine Ltd. (Nanjing, Jiangsu, China), while lysine was purchased from Beijing Solarbio Science and Technology Co. Ltd. (Beijing, China). RHL was synthesized at the Oncology Department of the Institute of Medicinal Biotechnology, Chinese Academy of Medical Sciences and Peking Union Medical College (Beijing, China; patent no. 2008100890258). Polyclonal rabbit anti-human Sirtuin 1 (SIRT1; 1:1,000; cat. no. 2493s), polyclonal rabbit anti-human p16 (1:1,000; cat. no. 4824), monoclonal rabbit anti-human p21 (1:1,000; cat. no. 2947s), monoclonal rabbit anti-human $\beta$-actin $(1: 1,000$; cat. no. 12620$)$ primary antibodies, and secondary antibodies against rabbit $(1: 5,000$; cat. no. 7074 s) or mouse (1:5,000; cat. no. 7076s) IgG were purchased from Cell Signaling Technology (Danvers, MA, USA). The prestained protein marker, p7708V, was purchased from New England Biolabs Ltd. (Beijing, China). All other chemicals were of standard analytical grade.

Animals and drug administration. A total of 40 male Kun-Ming mice (age, 7 weeks; weight, $32 \pm 2$ g) were purchased from the Institute of Laboratory Animal Science (Chinese Academy of Medical Sciences, Beijing, China). All procedures conducted with animals were approved by our institutional review board (Animal Experiments Ethics Board, Beijing Hospital, Beijing, China). The mice were housed and maintained at $22 \pm 2^{\circ} \mathrm{C}$ with $12 \mathrm{~h}$ light/dark cycles and a relative humidity of $40-60 \%$. Food was provided ad libitum throughout the study. Following one-week of acclimatization to the home cage, the mice were randomly divided into four groups, each comprising 10 mice, as follows: i) Model group, in which the mice were injected subcutaneously with D-gal at a dose of $100 \mathrm{mg} / \mathrm{kg} / \mathrm{day}$, and simultaneously given distilled water by intragastric gavage; ii) control group, in which the mice were injected subcutaneously with the same volume of normal saline, and simultaneously given distilled water by intragastric gavage; iii) RHL (25 mg/kg/day) group, in which the mice were injected subcutaneously with D-gal at a dose of $100 \mathrm{mg} / \mathrm{kg} /$ day, and simultaneously given RHL (25 mg/kg/day) by intragastric gavage; and iv) RHL (50 mg/kg/day) group, in which the mice were injected subcutaneously with D-gal at a dose of $100 \mathrm{mg} / \mathrm{kg} / \mathrm{day}$, and simultaneously given RHL $(50 \mathrm{mg} / \mathrm{kg} /$ day) by intragastric gavage. During the study, the performance and body weight of the mice were recorded every day. The mice were sacrificed following 8 weeks of treatment. Blood was collected through cardiac puncture and centrifuged at $1,500 \mathrm{x}$ g for $15 \mathrm{~min}$ at $4^{\circ} \mathrm{C}$ to obtain plasma. Plasma was stored at $-70^{\circ} \mathrm{C}$ until assays were performed. The liver and kidney tissues were immediately collected, weighed and homogenized $\left(4^{\circ} \mathrm{C} ; 3,000 \mathrm{x}\right.$ for $\left.15 \mathrm{~min}\right)$ for biochemical and histological analyses. The organ index was measured using the following equation: Organ index $(\mathrm{mg} / \mathrm{g})=$ organ weight (mg)/body weight (g) (26).

Biochemical and histological analysis. The antioxidant activities of superoxide dismutase (SOD) and glutathione peroxidase (GSH-Px), and the levels of malondialdehyde (MDA) in the blood and tissues were determined using SOD, GSH-Px and MDA kits, according to the manufacturer's instructions (Nanjing Jiancheng Bioengineering Institute, Nanjing, Jiangsu, China). Subsequent to fixing in $10 \%$ formalin for $24 \mathrm{~h}$, tissue samples were progressively dehydrated in different concentrations of ethanol, hyalinized in xylene, embedded in paraffin, sliced into thin sections $(5 \mu \mathrm{m})$, dewaxed and stained with hematoxylin and eosin (Beijing Solarbio Science and Technology Co. Ltd.). Sections were examined using an Olympus CK40 microscope (Olympus, Tokyo, Japan). Cross sections were selected from three plates per sample.

Western blot analysis. Western blot analysis was employed to detect the protein expression levels of SIRT1, p16 and p21. The tissues were treated with a lysis buffer and a mixture of phosphatase inhibitors (Roche, Indianapolis, IN, USA). Samples $(30 \mu \mathrm{g})$ were fractionated by $10 \%$ SDS-PAGE. Once the proteins were transferred to a polyvinylidene difluoride membrane, the membrane was incubated in a blocking buffer containing bovine serum albumin (1\%) and Tween-20 $(0.1 \%$ $\mathrm{v} / \mathrm{v}$ ) in phosphate-buffered saline at room temperature for $1 \mathrm{~h}$. The membrane was then incubated overnight at $4^{\circ} \mathrm{C}$ with the appropriate primary antibodies and then incubated with the appropriate secondary antibodies at room temperature for $2 \mathrm{~h}$. Each membrane was developed using an enhanced ChemiImager 5500 chemiluminescence system (Alpha Innotech Corporation, Miami, FL, USA).

Statistical analysis. Data are presented as the mean \pm standard deviation. Treatment effects were compared using the Student $t$-test and differences between the means were considered to be statistically significant when $\mathrm{P} \leq 0.05$.

\section{Results}

Behavior observations and organ index. No statistically significant differences $(\mathrm{P}>0.05)$ in food intake and body weight change were observed between the mice in the different groups (data not shown). Prior to being sacrificed, no mice died during the experimental procedure. Mice of the model group with D-gal demonstrated evident symptoms of aging, including slow movement, a lag in response, listlessness, and withered and lackluster fur. In addition, organ indexes of the liver and kidney of the model group were significantly lower $(\mathrm{P}<0.05)$ compared with those of the control group, as shown in Table I. However, RHL administration may improve those organ indexes $(\mathrm{P}<0.05)$.

Effects of RHL on the SOD activity in aging mice. The model group receiving D-gal showed significantly lower SOD activity $(\mathrm{P}<0.05)$ in the liver compared with that of the control mice. However, treatment of the aging mice with RHL significantly increased the SOD activity $(\mathrm{P}<0.05)$ following 8 weeks of treatment. In particular, the high-dose RHL group 
Table I. Organ index of mice in each group $(\mathrm{mg} / \mathrm{g})$.

\begin{tabular}{lcc}
\hline Group & Liver & Kidney \\
\hline Control & $46.86 \pm 5.43$ & $9.27 \pm 0.89$ \\
Model & $39.24 \pm 4.12^{\mathrm{a}}$ & $7.64 \pm 0.62^{\mathrm{a}}$ \\
RHL 25 $\mathrm{mg} / \mathrm{kg}$ & $44.76 \pm 4.89^{\mathrm{b}}$ & $8.34 \pm 0.66^{\mathrm{b}}$ \\
RHL $50 \mathrm{mg} / \mathrm{kg}$ & $45.64 \pm 3.65^{\mathrm{b}}$ & $9.01 \pm 0.78^{\mathrm{b}}$ \\
\hline
\end{tabular}

Values are presented as the mean \pm standard deviation. ${ }^{\mathrm{a}} \mathrm{P}<0.05$ vs. control group; ${ }^{\mathrm{b}}<0.05$ vs. model group. RHL, rhein lysinate.

demonstrated higher SOD activity than that of the model group $(\mathrm{P}<0.05)$. However, no statistically significant differences $(\mathrm{P}>0.05)$ were observed between the low- and high-dose RHL groups (Fig. 1). The results from the kidney and serum followed the same pattern.

Effects of RHL on the GSH-Px activity in aging mice. Mice of the model group receiving D-gal showed significantly lower activities of GSH-Px $(\mathrm{P}<0.05)$ in the liver compared with the control mice. However, treatment of the aging mice with RHL significantly increased the activities of GSH-Px $(\mathrm{P}<0.05)$ following 8 weeks of treatment (Fig. 2). The results from the kidney and serum were similar.

Effects of RHL on the MDA content in aging mice. The MDA content in the liver of the model group was significantly greater $(\mathrm{P}<0.05)$ than that of the control group, which indicated that the aging animal model had been successfully established. The MDA content of the RHL groups was much smaller $(\mathrm{P}<0.05)$ compared with that of the model group. In particular, the MDA content in the high-dose group was lower compared with that of the control group, but there were no statistically significant differences $(\mathrm{P}>0.05)$ between the low- and high-dose RHL groups (Fig. 3). Similar results were obtained for MDA in the kidney and serum.

Effects of RHL on liver and kidney morphological alterations. The morphological features of hematoxylin and eosin-stained liver sections are presented in Fig. 4. The hepatocytes of D-gal-treated mice exhibited extensive hepatic edema and some degrees of ballooning degeneration, and the cytoplasm color of hepatocytes became lighter as opposed to that of the control mice. Notably, RHL treatment was able to attenuate liver injury induced by D-gal in mice. The different doses of RHL demonstrated excellent liver-protecting activity, while hepatic edema was largely controlled. Similarly, the renal tubular epithelial cells of D-gal-treated mice exhibited edema (Fig. 5). The cell membrane penetrability of the epithelial cells was increased and the cytoplasm color became shallow and even transparent. Therefore, RHL may mitigate renal tubular edema, thus protecting the kidney.

Effects of RHL on the expression of proteins associated with aging. According to the results of western blot analysis, the expression levels of p16 and p21 increased in the liver and kidney $(\mathrm{P}<0.05)$, while SIRT1 expression decreased in

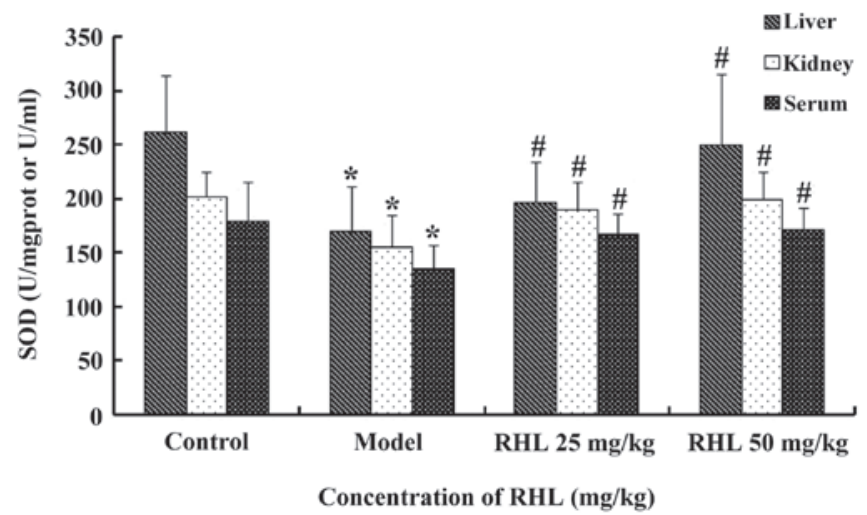

Figure 1. Effects of RHL on the SOD activity in aging mice. Values are presented as the mean \pm standard deviation. The model group mice were injected subcutaneously with $\mathrm{D}$-galactose. ${ }^{*} \mathrm{P}<0.05$ vs. control group; ${ }^{\#} \mathrm{P}<0.05$ vs. model group. RHL, rhein lysinate; SOD, superoxide dismutase.

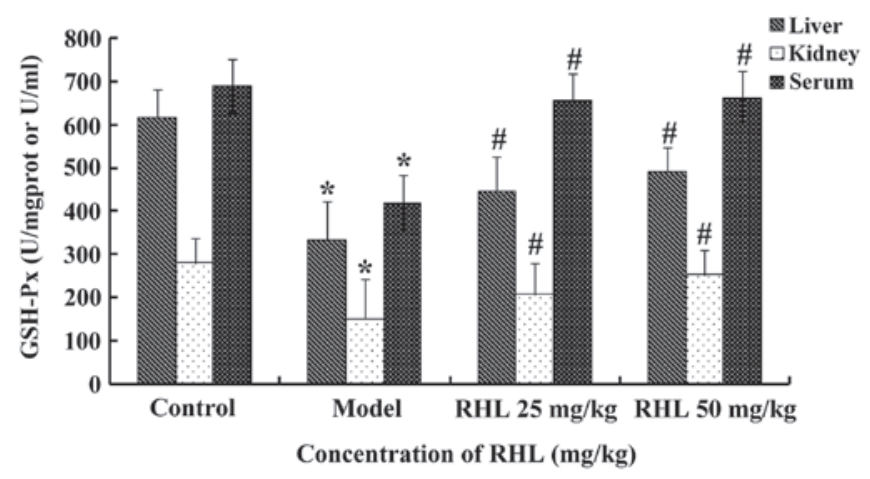

Figure 2. Effects of RHL on the GSH-Px activity in aging mice. Values are presented as the mean \pm standard deviation. The model group mice were injected subcutaneously with $\mathrm{D}$-galactose. ${ }^{*} \mathrm{P}<0.05$ vs. control group; ${ }^{\#} \mathrm{P}<0.05$ vs. model group. RHL, rhein lysinate; GSH-Px, glutathione peroxidase.

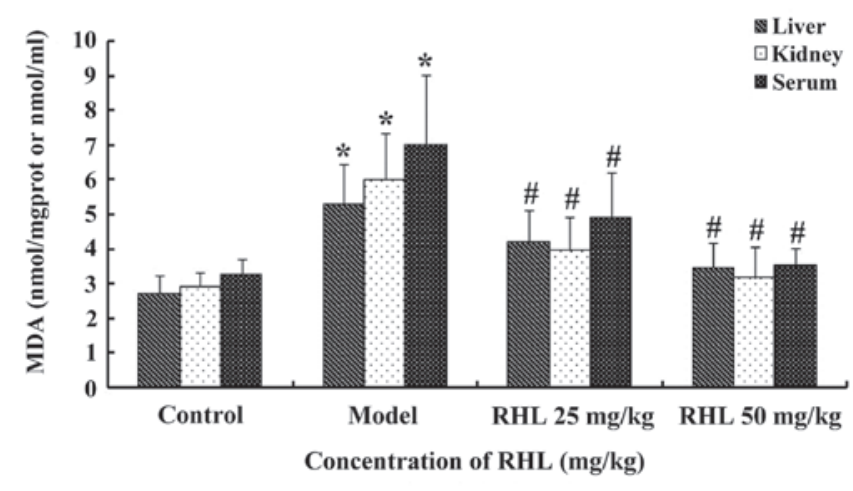

Figure 3. Effects of RHL on the MDA content in aging mice. Values are presented as the mean \pm standard deviation. The model group mice were injected subcutaneously with $\mathrm{D}$-galactose. ${ }^{*} \mathrm{P}<0.05$ vs. control group; ${ }^{\#} \mathrm{P}<0.05$ vs. model group. RHL, rhein lysinate; MDA, malondialdehyde.

D-gal-treated mice $(\mathrm{P}<0.05)$. Compared with the model group, the expression of p16 and p21, which was elevated in D-gal-treated mice, was inhibited in the $25 \mathrm{mg} / \mathrm{kg}$ and $50 \mathrm{mg} / \mathrm{kg}$ RHL groups $(\mathrm{P}<0.05)$ (Fig. 6). RHL also upregulated the level of SIRT1 expression compared with D-gal-treated mice $(\mathrm{P}<0.05)$. 


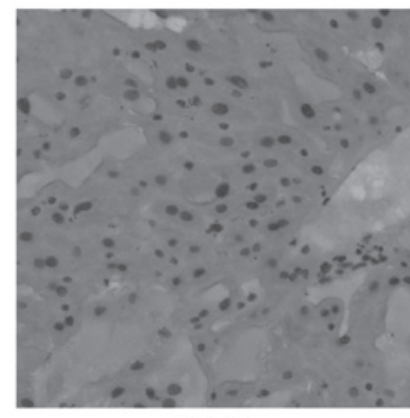

Con

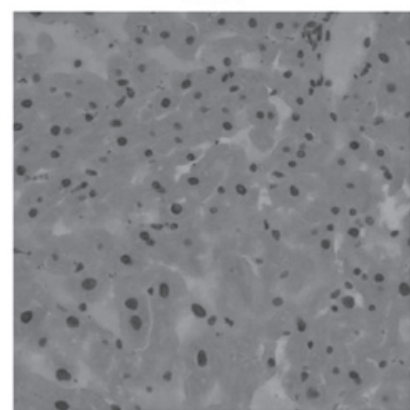

RHL(25)

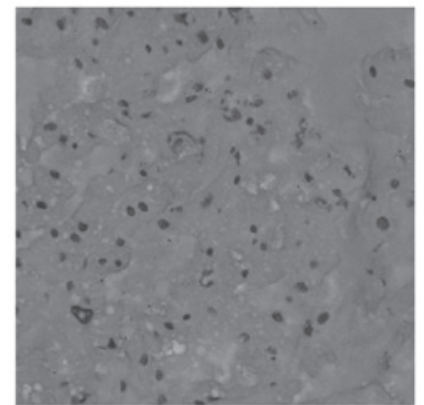

Model

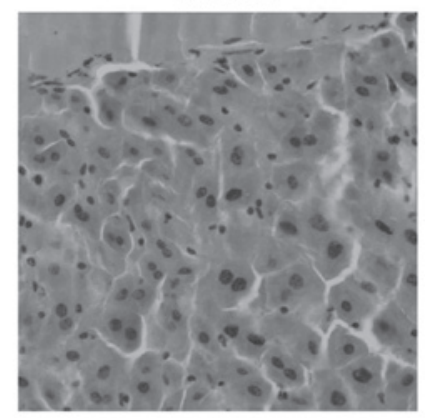

RHL(50)
Figure 4. Effect of RHL on the change of pathological profile in the liver of aging mice, as determined by hematoxylin and eosin staining (x200). The model group mice were injected subcutaneously with D-galactose. RHL(25), RHL 25 mg/kg group; RHL(50), 50 mg/kg group; RHL, rhein lysinate.

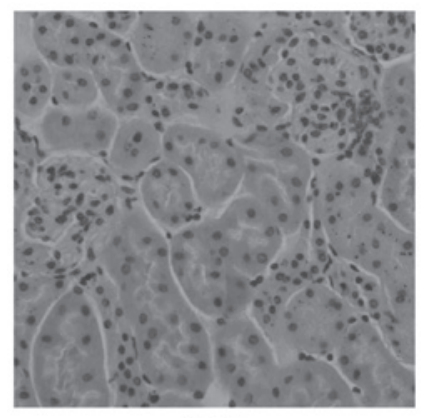

Con

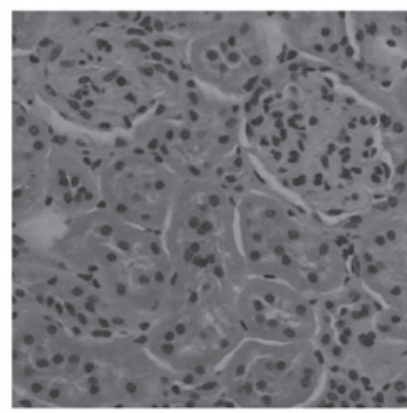

RHL(25)

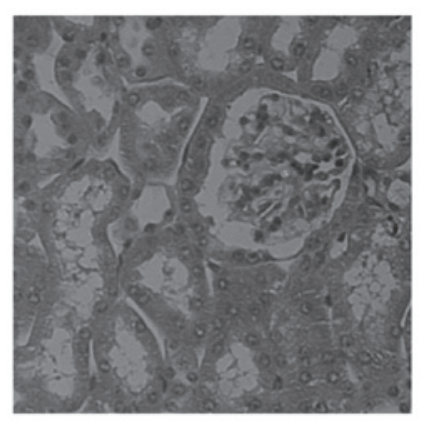

Model

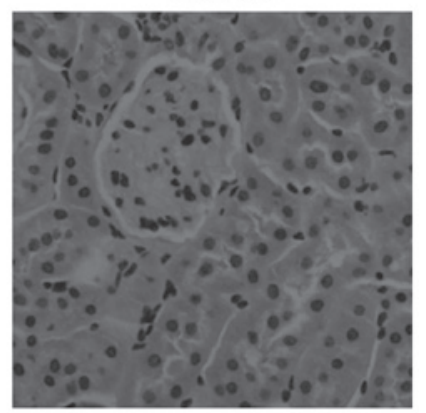

RHL(50)
Figure 5. Effect of RHL on the change of pathological profile in the kidney of aging mice, as determined by hematoxylin and eosin staining (x200). The model group mice were injected subcutaneously with D-galactose. RHL(25), RHL 25 mg/kg group; RHL(50), RHL 50 mg/kg group; RHL, rhein lysinate.

\section{Discussion}

From a biological perspective, aging is an inevitable spontaneous process and a complicated natural phenomenon $(27,28)$.
A
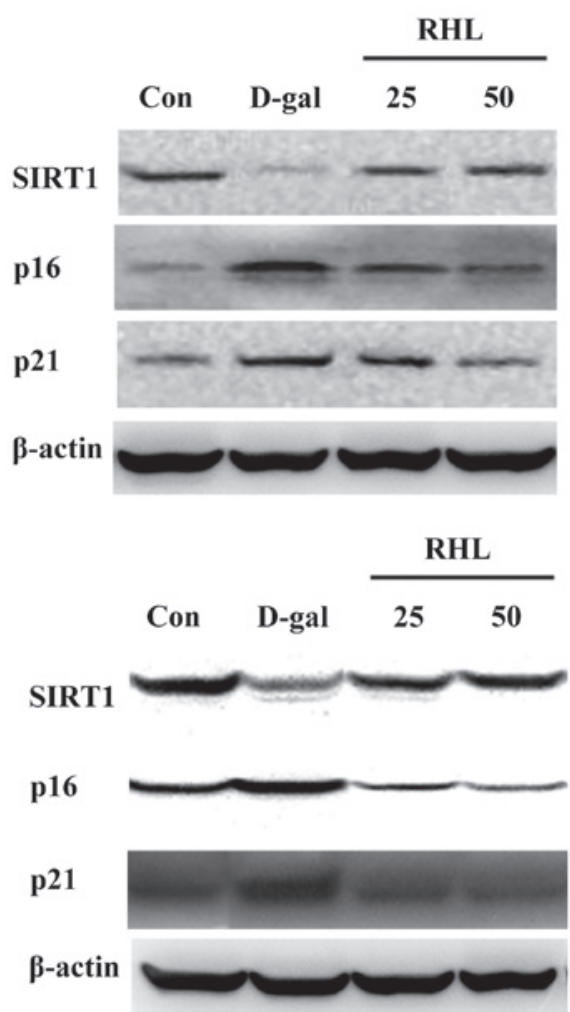

Figure 6. Effect of RHL on the expression of proteins associated with aging-related genes in the (A) liver and (B) kidney were are analyzed by Western blotting. $\beta$-actin was used as the internal control. Con, normal mice; D-gal, model group (injected subcutaneously with D-gal); RHL 25, RHL $25 \mathrm{mg} / \mathrm{kg}$ group; RHL 50, RHL $50 \mathrm{mg} / \mathrm{kg}$ group; RHL, rhein lysinate; D-gal, D-galactose.

Although certain mechanisms of aging have been proposed, theories linking aging and cellular oxidative stress have received more support $(29,30)$.

With the improvement of living standards all over the world, individuals are increasingly concerned about their appearance and health (3). Postponing body aging has become a key topic of concern for numerous people (3). The processes that delay and/or reverse visible signs of aging are termed as anti-aging. Numerous scientists and pharmaceutical companies have attempted to develop drugs to reduce the speed of human aging, but no effective drug has been discovered to date. In the last decade, the importance of folk medicine and herbal medicines has been revisited and has resulted in the development of various effective drugs for anti-aging. The majority of anti-aging herbs have antioxidant components and reduce free radicals which are by-products of abnormal body metabolism in the elderly.

Rhein, one of the major bioactive constituents of the rhizome of rhubarb (Rheum palmatum Linn. or R. tanguticum Maxim), has received attention for its anti-aging effects in vitro (25). RHL is a novel compound, which was synthesized by our team. Compared with rhein, RHL is easily dissolved in water $(25,31)$.

In the present study, to the best of our knowledge, the effects of RHL on oxidative stress and aging-associated gene expression have been investigated for the first time in an animal model, using chronic administration of D-gal to induce 
aging. The model groups in the experiments demonstrated clear differences with other groups in their daily behavior, pathological sections and biochemical indexes. In addition, no mouse succumbed due to the D-gal model; therefore, the aging model was created successfully. The results revealed a significant anti-aging effect for RHL in D-gal-induced aging mice.

The kidney and liver are important organs for detoxification; however, their functions gradually decline due to age-associated structural atrophy (12). The present results indicated that the kidney and liver were atrophied in D-gal-induced aging mice. However, RHL may be able to increase these organ indexes.

Aging model mice are characterized by an increased concentration of ROS and a significant reduction in their antioxidant defenses (32). ROS are chemically reactive oxygen-derived molecules. A growing body of evidence suggests that accumulation of ROS in biological systems causes oxidative damage to tissues, affecting cellular integrity and function. Oxidative damage caused by ROS has frequently been associated with the pathogenesis of various diseases, and ROS are considered to be important causative factors in the aging process (33).

Free radicals derived from oxygen exert detrimental effects on humans, including peroxidation of membrane lipids, enzyme inactivation, DNA fragmentation and activation of apoptosis (34). MDA is a major biomarker that is observed during the final stages of lipid peroxidation initiated by excessive ROS. In addition, supplementation with antioxidants has been reported to be beneficial with respect to slowing down the aging process (35).

As part of the antioxidant defense systems, a group of enzymes, including SOD and GSH-Px, function as superoxide anion and hydrogen peroxide scavengers to prevent ROS-induced damage. The MDA, SOD and GSH-Px levels are, therefore, indicators of oxidative stress status. The present results indicated that RHL markedly diminished oxidative stress in the aged mice by increasing the activities of SOD and GSH-Px in the liver, kidney and serum and decreasing the content of MDA, supporting the mechanism of action of RHL and the theory of oxidative stress in aging. Thus, it can be deduced that RHL inhibits aging partly by reducing the level of ROS.

The expression levels of SIRT1, p21 and p16 are closely associated with mammalian aging. SIRT1 can increase deacetylation of p53 and SIRT1 is a nicotinamide adenine dinucleotide-dependent deacetylase that slows aging in lower organisms and inhibits the development of aging-associated diseases in mammals. SIRT1 affects a variety of biological functions, including DNA repair, energy metabolism, tumor suppression and mitochondrial homeostasis. There is increasing evidence that elevated SIRT1 activity can have beneficial effects on aging and aging-associated diseases in mammals (36-38). These effects may be associated with SIRT1-mediated modulation of DNA and metabolic damage $(36,37)$. Roles for SIRT1 in preventing endothelial cells from replicative senescence or stress-induced premature senescence have been reported in previous years $(39,40)$. These anti-aging effects were associated with the effects of deacetylation of liver kinase B1 or p53 by SIRT1. SIRT1 was also demonstrated to protect human umbilical cord fibroblasts from replicative senescence by promoting the transcription of telomerase reverse transcriptase (41).

The p16 gene, a cyclin-dependent kinase inhibitor, is considered to play an important role in tumor growth suppression and cell senescence $(42,43)$. Expression of p16 notably increases with aging in the majority of rodents and in human tissues. The accumulation of p16 contributes to senescence by negatively regulating the cell cycle in vitro and in vivo (44-46).

Another inhibitor of cell cycle progression, p21, increases with age and contributes to the impaired cellular regeneration of an aging organism. p21 deficiency partially prevented age-induced decline in cell proliferation and tissue function (47). In the present study, RHL was found to markedly decrease p16 and p21 expression levels and increase SIRT1 expression. These results suggested that RHL may modulate age-associated gene expression.

In conclusion, the results obtained in the current study demonstrated that RHL can reduce the aging effects induced by $\mathrm{D}$-gal injection in mice. This effect may be mediated, at least partly, through enhancing antioxidant activity, scavenging free radicals and modulating aging-associated gene expression. These data suggest that RHL has anti-aging effects, and has development potential as an anti-aging medicine.

\section{Acknowledgements}

The present study was supported by grants from the National Natural Science Foundation of China (no. 81001439) and the General Program of Natural Science Foundation of Hebei Province of China (no. H2012401030).

\section{References}

1. Balaban RS, Nemoto S and Finkel T: Mitochondria, oxidants and aging. Cell 120: 483-495, 2005.

2. Peiris H, Dubach D, Jessup CF, Unterweger P, Raghupathi R, Muyderman H, Zanin MP, Mackenzie K, Pritchard MA and Keating DJ: RCAN1 regulates mitochondrial function and increases susceptibility to oxidative stress in mammalian cells. Oxid Med Cell Longev 2014: 520316, 2014.

3. Rattan SI: Aging is not a disease: Implications for intervention. Aging Dis 5: 196-202, 2014.

4. Butterfield DA, Di Domenico F and Barone E: Elevated risk of type 2 diabetes for development of Alzheimer disease: A key role for oxidative stress in brain. Biochim Biophys Acta 1842: 1693-1706, 2014.

5. Yu W, Bonnet M, Farso M, Ma K, Chabot JG, Martin E, Torriglia A, Guan Z, McLaurin J, Quirion R and Krantic S: The expression of apoptosis inducing factor (AIF) is associated with aging-related cell death in the cortex but not in the hippocampus in the TgCRND8 mouse model of Alzheimer's disease. BMC Neurosci 15: 73, 2014.

6. Jo-Watanabe A, Ohse T, Nishimatsu H, Takahashi M, Ikeda Y, Wada T, Shirakawa J, Nagai R, Miyata T, Nagano T, Hirata Y, et al: Glyoxalase I reduces glycative and oxidative stress and prevents age-related endothelial dysfunction through modulation of endothelial nitric oxide synthase phosphorylation. Aging Cell 13: 519-528, 2014

7. Jomova K, Vondrakova D, Lawson M and Valko M: Metals, oxidative stress and neurodegenerative disorders. Mol Cell Biochem 345: 91-104, 2010.

8. Jomova $\mathrm{K}$ and Valko M: Advances in metal-induced oxidative stress and human disease. Toxicology 283: 65-87, 2011.

9. Chen B,Zhong Y, Peng W, Sun Y and Kong WJ: Age-related changes in the central auditory system: Comparison of D-galactose-induced aging rats and naturally aging rats. Brain Res 1344: 43-53, 2010.

10. Li L, Ng TB, Gao W, Li W, Fu M, Niu SM, Zhao L, Chen RR and Liu F: Antioxidant activity of gallic acid from rose flowers in senescence accelerated mice. Life Sci 77: 230-240, 2005. 
11. Chen HL, Wang $\mathrm{CH}$, Kuo YW and Tsai CH: Antioxidative and hepatoprotective effects of fructo-oligosaccharide in D-galactose-treated Balb/cJ mice. Br J Nutr 105: 805-809, 2011

12. Ye Y, Jia RR, Tang L and Chen F: In vivo antioxidant and anti-skin aging activities of ethyl acetate extraction from idesia polycarpa defatted fruit residue in aging mice induced by D-galactose. Evid Based Complement Alternat Med 2014: $185716,2014$.

13. Li YN, Guo Y, Xi MM, Yang P, Zhou XY, Yin S, Hai CX, Li JG and Qin XJ: Saponins from Aralia taibaiensis attenuate $\mathrm{D}$-galactose-induced aging in rats by activating FOXO3a and Nrf2 pathways. Oxid Med Cell Longev 2014: 320513, 2014.

14. Cui X, Zuo P, Zhang Q, Li X, Hu Y, Long J, Packer L and Liu J: Chronic systemic D-galactose exposure induces memory loss, neurodegeneration and oxidative damage in mice: Protective effects of R-alpha-lipoic acid. J Neurosci Res 84: 647-654, 2006

15. Cui X, Wang L, Zuo P, Han Z, Fang Z, Li W and Liu J: D-galactose-caused life shortening in Drosophila melanogaster and Musca domestica is associated with oxidative stress. Biogerontology 5: 317-325, 2004.

16. Zhang ZF, Fan SH, Zheng YL, Lu J, Wu DM, Shan Q and Hu B: Purple sweet potato color attenuates oxidative stress and inflammatory response induced by D-galactose in mouse liver. Food Chem Toxicol 47: 496-501, 2009.

17. Zhong SZ, Ge QH, Qu R, Li Q and Ma SP: Paeonol attenuates neurotoxicity and ameliorates cognitive impairment induced by D-galactose in ICR mice. J Neurol Sci 277: 58-64, 2009.

18. Mao J, Huang S, Liu S, Feng XL, Yu M, Liu J, Sun YE, Chen G, Yu Y, Zhao J and Pei G: A herbal medicine for Alzheimer's disease and its active constituents promote neural progenitor proliferation. Aging Cell 2015 May 25 doi: 10.1111/acel.12356. [Epub ahead of print].

19. Rahimi R and Abdollahi M.Herbal medicines for the management of irritable bowel syndrome: A comprehensive review. World J Gastroenterol 18: 589-600, 2012.

20. Wang $M$ and Lei Y: Delaying vascular aging with Chinese medicine: Implications from an overview of the p53 and miR-34s family. Chin J Integr Med 17: 635-639, 2011.

21. Xiong D, Yu LX, Yan X, Guo C and Xiong Y: Effects of root and stem extracts of Asparagus cochinchinensis on biochemical indicators related to aging in the brain and liver of mice. Am J Chin Med 39: 719-726, 2011.

22. Du H, Shao J, Gu P, Lu B, Ye X and Liu Z: Improvement of glucose tolerance by rhein with restored early-phase insulin secretion in $\mathrm{db} / \mathrm{db}$ mice. J Endocrinol Invest 35: 607-612, 2012.

23. Malaguti C, Vilella CA, Vieira KP, Souza GH, Hyslop S and Zollner Rde L: Diacerhein downregulate proinflammatory cytokines expression and decrease the autoimmune diabetes frequency in nonobese diabetic (NOD) mice. Int Immunopharmacol 8: 782-791, 2008.

24. Martin G, Bogdanowicz P, Domagala F, Ficheux H and Pujol JP: Articular chondrocytes cultured in hypoxia: Their response to interleukin-1beta and rhein, the active metabolite of diacerhein. Biorheology 41: 549-561, 2004.

25. Lin YJ, Zhen YZ, Wei J, Liu B, Yu ZY and Hu G: Effects of Rhein lysinate on $\mathrm{H}_{2} \mathrm{O}_{2}$-induced cellular senescence of human umbilical vascular endothelial cells. Acta Pharmacol Sin 32. 1246-1252, 2011.

26. Sun JH, Liu YM, Cao T and Ouyang WQ: Effect of kinetin on ovary and uterus in D-galactose-induced female mouse model of aging. Sheng Li Xue Bao 65: 389-394, 2013 (In Chinese).

27. Li Z, Liu R, Kang X and Wang X: Study on establishment of kidney deficient aging model and comparison with D-galactose induced aging model. Zhongguo Zhong Yao Za Zhi 37: 2435-2438, 2012 (In Chinese)

28. Prisila Dulcy C, Singh HK, Preethi J and Rajan KE: Standardized extract of Bacopa monniera (BESEB CDRI-08) attenuates contextual associative learning deficits in the aging rat's brain induced by D-galactose. J Neurosci Res 90: 2053-2064, 2012.
29. Mukherjee A and Haldar C: Melatonin membrane receptor (MT1R) expression and nitro-oxidative stress in testis of golden hamster, Mesocricetus auratus: An age-dependent study. Exp Gerontol 69: 211-220, 2015.

30. Tatone C, Di Emidio G, Vitti M, Di Carlo M, Santini S Jr, D'Alessandro AM, Falone S and Amicarelli F: Sirtuin functions in female fertility: Possible role in oxidative stress and aging. Oxid Med Cell Longev 2015: 659687, 2015.

31. Hu G1, Liu J, Zhen YZ, Xu R, Qiao Y, Wei J, Tu P, Lin YJ. Rhein lysinate increases the median survival time of SAMP10 mice: Protective role in the kidney. Acta Pharmacol Sin 34: 515-521, 2013.

32. Nasto LA, Robinson AR, Ngo K, Clauson CL, Dong Q, St Croix C, Sowa G, Pola E, Robbins PD, Kang J, et al: Mitochondrial-derived reactive oxygen species (ROS) play a causal role in aging-related intervertebral disc degeneration. J Orthop Res 31: 1150-1157, 2013.

33. Datta HS, Mitra SK and Patwardhan B: Wound healing activity of topical application forms based on Ayurveda. Evid Based Complement Alternat Med 2011: 134378, 2011.

34. Speakman JR and Selman C: The free-radical damage theory: Accumulating evidence against a simple link of oxidative stress to ageing and lifespan. Bioessays 33: 255-259, 2011.

35. Koyama H, Nojiri H, Kawakami S, Sunagawa T, Shirasawa $\mathrm{T}$ and Shimizu T. Antioxidants improve the phenotypes of dilated cardiomyopathy and muscle fatigue in mitochondrial superoxide dismutase-deficient mice. Molecules 18: 1383-1393, 2013.

36. Herranz D and Serrano M: Impact of Sirt1 on mammalian aging. Aging (Albany NY) 2: 315-316, 2010.

37. Herranz D, Muñoz-Martin M, Cañamero M, Mulero F, Martinez-Pastor B, Fernandez-Capetillo O and Serrano M: Sirt1 improves healthy ageing and protects from metabolic syndrome-associated cancer. Nat Commun 1: 3, 2010.

38. Herskovits AZ and Guarente L: SIRT1 in neurodevelopment and brain senescence. Neuron 81: 471-483, 2014.

39. Ota H, Akishita M, Eto M, Iijima K, Kaneki M and Ouchi Y: Sirt1 modulates premature senescence-like phenotype in human endothelial cells. J Mol Cell Cardiol 43: 571-579, 2007.

40. Zu Y, Liu L, Lee MY, Xu C, Liang Y, Man RY, Vanhoutte PM and Wang Y: SIRT1 promotes proliferation and prevents senescence through targeting LKB1 in primary porcine aortic endothelial cells. Circ Res 106: 1384-1393, 2010.

41. Yamashita S, Ogawa K, Ikei T, Udono M, Fujiki $T$ and Katakura Y: SIRT1 prevents replicative senescence of normal human umbilical cord fibroblast through potentiating the transcription of human telomerase reverse transcriptase gene. Biochem Biophys Res Commun 417: 630-634, 2012.

42. Gil J and Peters G: Regulation of the INK4b-ARF-INK4a tumour suppressor locus: All for one or one for all. Nat Rev Mol Cell Biol 7: 667-77, 2006.

43. Krishnamurthy J, Torrice C, Ramsey MR, Kovalev GI, Al-Regaiey K, Su L and Sharpless NE: Ink4a/Arf expression is a biomarker of aging. J Clin Invest 114: 1299-1307, 2004.

44. Alcorta DA, Xiong Y, Phelps D, Hannon G, Beach D and Barrett JC: Involvement of the cyclin-dependent kinase inhibitor p16 (INK4a) in replicative senescence of normal human fibroblasts. Proc Natl Acad Sci 93: 13742-13747, 1996.

45. Melk A, Schmidt BM, Takeuchi O, Sawitzki B, Rayner DC and Halloran PF: Expression of p16INK4a and other cell cycle regulator and senescence associated genes in aging human kidney. Kidney Int 65: 510-520, 2004.

46. Zhang X, Wu X, Tang W and Luo Y: Loss of p16 (Ink4a) function rescues cellular senescence induced by telomere dysfunction. Int J Mol Sci 13: 5866-5877, 2012.

47. Li Y and Tollefsbol TO: p16 (INK4a) suppression by glucose restriction contributes to human cellular lifespan extension through SIRT1-mediated epigenetic and genetic mechanisms. PLoS One 6: e17421, 2011. 